
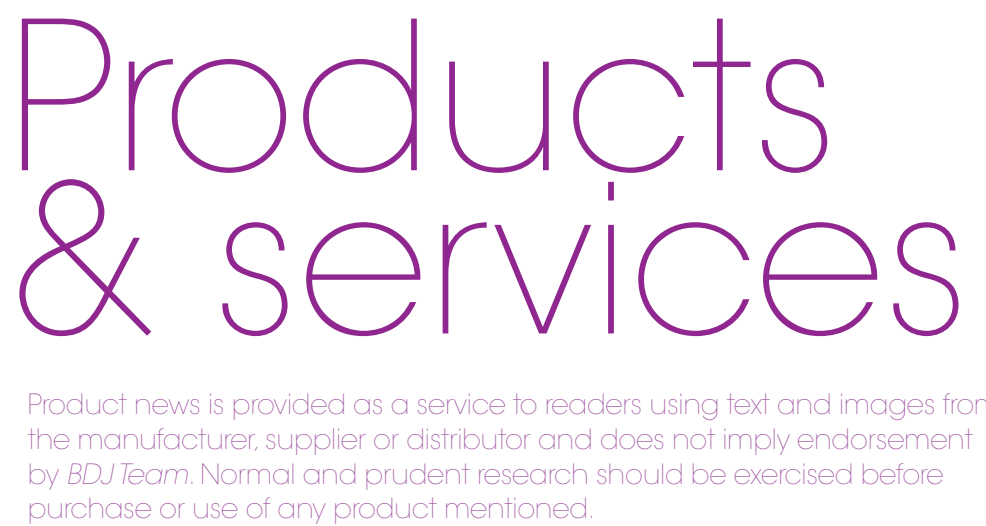

\title{
WIN A PREMIUM GIFT BOX WITH ULTRADEX
}

Which of the following words cannot be found in this grid?

PROTECTS

RESTORES

ELIMINATES

VSC

PLAQUE BIOFILM

TWELVE HOURS

FRESH BREATH

LOW ABRASION

ORAL RINSE

TOOTHPASTE

TOOTHBRUSH

RECALCIFYING

ULTRADEX

PERIPRODUCTS

WHITENING

ORAL CARE

The award-winning

Performance Oral Care brand UltraDEX is giving five lucky $B D J$ Team readers the chance to win one of its premium gift boxes. To be in with a chance of winning, complete the Word Search. One of the words in the list above cannot be found in the grid - email this word to bdjteam@nature.com with your name, job title and postal address.

UltraDEX bridges the gap between professional and home care. Backed by

extensive scientific research and powered by stabilised chlorine dioxide, UltraDEX helps to PROTECT against plaque, RESTORES natural whiteness by gently oxidising stains and instantly ELIMINATES bad breath compounds (VSCs) for 12 hours. Free from alcohol, gluten, harsh abrasives and sodium

$$
\begin{aligned}
& \text { O F J }
\end{aligned}
$$

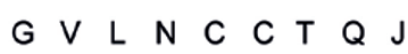

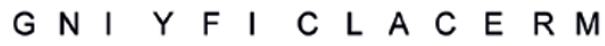

$$
\begin{aligned}
& \text { C Z D K P E R I P R O D U C T S J } \\
& T H \text { W E I W W K O W F U W A J A U M H } \\
& \text { Y A S N P D O V N G M R V E R A C L A R O } \\
& \text { E N K N S U P F C R R U E R A R H X C D } Q
\end{aligned}
$$

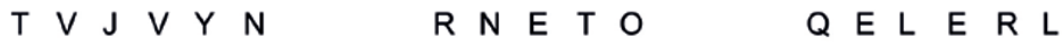

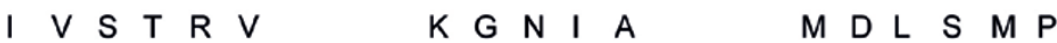

A M K V B E H N C S E T A N I M I L E A F B Q O T W I $Q H E S P G E M M Q S V U S P G S R E L W P H$

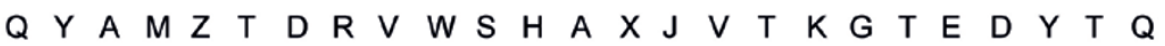

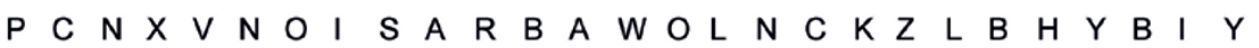
$M L V O G O R \quad C K H V M C D N P A B S N U N N$ W S Y A G A A E Y X E J S M X T D F R E S H B R E A T H N K Q

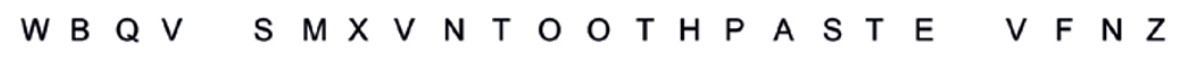
$X S I U U$ O E S N I R L A R O G I $X \quad C \quad C \quad T \quad J \quad G$ $S H B E E N \quad I H E L T P U Q L D N \quad N$ W $~ N A$

$$
T D M C B \quad R E \text { B I } F
$$

$Z C D O C$ I

$P \quad L \quad N \times G$

K E W C G O M Z N M C N I E D S V L E M Y Q G T Q H L F C L W O U L V W E J T H L R

L $P$ O

$$
\begin{aligned}
& \text { B Q R R I O L P Y D O O Q } \\
& X \text { P T } V \text { E E M S A U B W G L } \\
& \text { O } K V \text { V F T R C I N } \\
& \text { D S L }
\end{aligned}
$$

lauryl sulphate, the UltraDEX range is gentle, effective and safe for long term daily use. The rinses, toothpastes and spray are approved by the Vegetarian Society, registered with the Vegan Society and have Kosher status.

Each UltraDEX Oral Hygiene gift box contains the alcohol-free Daily Oral Rinse, SLS free Low-Abrasion Toothpaste, Fresh Breath Oral Spray, Tongue Cleanser, hyG Ionic Toothbrush, the Recalcifying \& Whitening Toothpaste and Oral Rinse, Interdental Tape and Wire-Free Interdental Brushes. For more information visit www. periproducts.co.uk. 